\title{
Téoros
}

Revue de recherche en tourisme

\section{L'utilisation du label UNESCO dans la communication touristique}

\section{Entre choix et contrainte}

\section{Luc Florent}

Volume 30, numéro 2, 2011

Tourisme et patrimoine mondial

URI : https://id.erudit.org/iderudit/1012238ar

DOI : https://doi.org/10.7202/1012238ar

Aller au sommaire du numéro

Éditeur(s)

Université du Québec à Montréal

ISSN

0712-8657 (imprimé)

1923-2705 (numérique)

Découvrir la revue

Citer cet article

Florent, L. (2011). L’utilisation du label UNESCO dans la communication touristique : entre choix et contrainte. Téoros, 30(2), 17-27. https://doi.org/10.7202/1012238ar
Résumé de l'article

Lorsqu'elle a institué la liste du patrimoine mondial, l'UNESCO cherchait avant tout à protéger et valoriser les sites naturels et culturels, biens communs de l'humanité. Si cette fonction reste toujours la préoccupation première, force est de constater que le label " patrimoine mondial de l'humanité » est de plus en plus utilisé comme un argument commercial traduisant la qualité du site. On peut donc se demander comment les touristes perçoivent ce label et comment ils l'utilisent dans le choix de leur destination de séjour touristique. Par un travail d'enquête, nous montrons qu'il est un élément déterminant dans le processus de choix. Nous avons ensuite observé comment les collectivités locales utilisaient ce label dans leur communication Internet et si elles avaient conscience de l'intérêt du public. On constate alors que si certaines peuvent s'en passer, d'autres ont l'impérieuse nécessité de l'utiliser pour augmenter leur attractivité. 


\title{
L'utilisation du label UNESCO dans la communication touristique Entre choix et contrainte
}

\author{
Luc FLORENT \\ Docteur en géographie \\ Enseignant-chercheur \\ Groupe ESC Troyes, École Supérieure de Commerce de Troyes \\ Luc.florent@groupe-esc-troyes.com
}

\begin{abstract}
RÉSUMÉ: Lorsqu'elle a institué la liste du patrimoine mondial, I'UNESCO cherchait avant tout à protéger et valoriser les sites naturels et culturels, biens communs de l'humanité. Si cette fonction reste toujours la préoccupation première, force est de constater que le label "patrimoine mondial de l'humanité» est de plus en plus utilisé comme un argument commercial traduisant la qualité du site. On peut donc se demander comment les touristes perçoivent ce label et comment ils l'utilisent dans le choix de leur destination de séjour touristique. Par un travail d'enquête, nous montrons qu'il est un élément déterminant dans le processus de choix. Nous avons ensuite observé comment les collectivités locales utilisaient ce label dans leur communication Internet et si elles avaient conscience de l'intérêt du public. On constate alors que si certaines peuvent s'en passer, d'autres ont l'impérieuse nécessité de l'utiliser pour augmenter leur attractivité.
\end{abstract}

Mots-clés: Label, patrimoine mondial, communication, collectivités locales, attractivité.

Les labels concernent désormais tous les types de produits : du camembert à la voiture en passant par les services bancaires et les produits touristiques.

Historiquement, la labellisation avait pour objectif de rassurer sur la qualité (Chameroyet Chandon, 2010). Cette fonction domine puisqu'il s'agit toujours d'attribuer au produit ou au service un signe crédible qui informe sur les dimensions de qualité et qui émane d'un organisme différent de l'organisme propriétaire (Larceneux, 2003a). Cependant, il s'est ajouté à cela une volonté de contrôler la fraude, une protection sanitaire, une dimension marketing et commerciale. La plupart des études menées sur le sujet montre que les labels ont des impacts sur les stratégies d'achat des consommateurs dans la mesure où ils les rassurent sur la qualité du produit (Chameroy et Chandon, 2010).

Le tourisme n'échappe pas à la règle et il n'est pas en retard dans cette pratique. Ainsi le guide Michelin donne depuis le début du $\mathrm{XX}^{\mathrm{e}}$ siècle des indications sur les sites touristiques et, depuis les années 1920, des étoiles aux meilleurs restaurants. Cette stratégie de labellisation touche désormais l'ensemble du monde touristique et s'étend au patrimoine. Les communes cherchent à obtenir les labels de "ville d'art et d'histoire", de «plus beau village de France», de «ville fleurie» et aussi, pour certains biens, de "patrimoine mondial de l'humanité». Chaque année, elles sont plus nombreuses à tenter d'obtenir cette qualification dont elles supposent qu'elle augmentera la fréquentation touristique.

Dans la mesure où les biens inscrits sur la liste du patrimoine mondial de l'UNESCO sont très médiatisés et l'appellation très convoitée par les collectivités locales, l'étude est centrée sur label UNESCO en France. L'objectif est de vérifier que ce label UNESCOest facteur d'attractivité et qu'il a un impact sur la consommation touristique et, par la suite, de voir comment les acteurs bénéficiant de ce label l'utilisaient dans leurs communications touristiques. En effet, si, comme nous le supposons, le label UNESCO est déclencheur de voyage, les " propriétaires » de biens inscrits auraient tout intérêt à mettre fortement en avant l'inscription. Par une étude de terrain réalisée en France, à Provins, Avignon et Besançon, l'auteur a observé les pratiques actuelles en la matière. Il est apparu nécessaire de mener une nouvelle étude sur la question de l'impact des labels sur le choix d'une destination. À la différence des travaux précédemment cités, l'étude est centrée sur un seul de ces labels, le label UNESCO car il s'agit de mesurer l'impact de ce label en particulier (nous parlerons de label UNESCO, mais il s'agit en réalité des sites inscrits sur la 
liste du patrimoine mondial que souvent, par raccourci, on qualifie de «site UNESCO»). En effet, pour beaucoup, il est censé augmenter l'attractivité touristique du site inscrit, et les nombreuses tentatives des acteurs locaux pour l'obtenir témoignent de cet intérêt supposé. L'attractivité touristique est la capacité à attirer les touristes sur son territoire. Elle repose sur des attractions touristiques, c'est-à-dire tous les éléments localisés ailleurs qui attirent le voyageur en dehors de son territoire (Lew, 1987). Plus précisément, ce sont les éléments tangibles et intangibles qui, à partir d'une mise en valeur appropriée de ressources naturelles et culturelles, auraient la capacité d'attirer les touristes (MacCannell, 1989, cité par de Grandpré, 2007). Pour Leiper(1990 : 370-382), les attractions sont composées d'un objet, d'un marqueur et d'un touriste susceptible d'être attiré. La problématique est ici de vérifier que le label UNESCO est bien un marqueur et qu'il contribue à transformer une ressource naturelle ou culturelle en une attraction touristique. En un mot, le label UNESCO est-il facteur d'attractivité? L'auteur du présent article pense à priori que c'est le cas et tentera de le démontrer.

Pour cela, nous présenterons d'abord l'état de la recherche sur la question de l'impact des labels sur la consommation touristique. Nous nous intéresserons ensuite au cas particulier du label UNESCO en présentant les résultats d'une enquête réalisée sur plusieurs sites UNESCO. Nous terminerons enfin par décrire la manière dont les acteurs utilisent le label UNESCO dans leur communication pour dégager les logiques en cours.

\section{Image, label et choix de destination touristique}

Le choix d'une destination touristique reste un processus complexe. Il l'est d'autant plus qu'aujourd'hui les offres de destination se sont multipliées. Certains ont montré que les frontières du tourisme sont quasiment inexistantes (Stocket al., 2003 : 143) et que globalement on peut faire du tourisme partout. Plusieurs paramètres interviennent dans le processus de choix. Ainsi une étude américaine citée par Frochot et Legohérel (2007 : 43) montre que le but du voyage, les partenaires, le temps et le budget disponibles, la période de l'année, les tarifs et les sources d'informations sont pris en compte par le touriste pour choisir son lieu de voyage. C'est en fonction du type de vacances souhaité et de tous ces paramètres que l'on décide de partir ici ou ailleurs. Finalement les destinations possibles seraient assez peu nombreuses, pas plus de sept d'après Moutinho (1987).

L'arbitrage entre ces quelques lieux de vacances va se faire sur l'image du territoire. Il semble en effet que ce soit le critère fondamental de décision, comme l'ont montré plusieurs études (Pike, 2002;Walmsley et Young, 1998), et un outil d'étude essentiel dans nos disciplines, comme l'a montré Knafou (1992: 859) :

La force de l'image dans le champ du tourisme n'est plus à démontrer. Elle explique qu'un des développements importants de la géographie du tourisme se fasse en liaison avec les approches et les problématiques de la géographie des représentations. Il est impossible de vouloir comprendre le fonctionnement des espaces touristiques si on ne s'attache pas, à la fois, aux images qu'ils produisent $[. .$.$] et aux pratiques induites par l'ensemble$ des images relatives au tourisme et aux loisirs, à l'origine des différentes perceptions du territoire touristique.

La construction de l'image territoriale est aussi un processus complexe. Chaque lieu dispose d'éléments matériels (dont le climat, les infrastructures touristiques, les transports oule patrimoine) et idéels (dont la culture, la tradition oul'histoire) qui renvoient une certaine image du territoire. Celle-ci est perturbée par la communication touristique et les caractéristiques socioculturelles du touriste. Celui-ci, avant de choisir sa destination, se renseigne et se documente sur les différents territoires qu'il pourrait fréquenter. Il y a donc une interprétation du territoire qui s'effectue avant même le début des vacances. Il imagine les paysages et les sites qu'il va découvrir. En réalité, il se fait une image du territoirequ'il va traverser comme le montreGuy Di Méo (1998 : 31) citant Marcel Proust qui confie dans Du côté de chez Swann : [Q] $[\mathrm{Q}]$ and je pensais à Florence, c'était comme une ville miraculeusement embaumée et semblable à une corolle, parce qu'elle s'appelait la cité des lys et sa cathédrale, Sainte-Marie-des-Fleurs.»

La cognition est caractérisée à la fois par les représentations mentales et par les traitements réalisés sur celles-ci. Ceux-ci s'opèrent sur les représentations initiales et génèrent de nouvelles représentations qui seront à leur tour l'objet de traitements ultérieurs. Les traitements peuvent se fonder sur ce qui est observé au cours du séjour : paysage, patrimoine, accueil, hébergement, etc. C'est ainsi que se constitue l'image finale du territoire(voir illustration 1).

Cette image finale est fondamentale pour les territoires, car elle va influer sur les intentions de retour et sur l'appropriation de ce territoire. Hall et Page (1999 : 209) soulignent que "[t]he mental image of a place [is]an individual's own view of reality, but [it is] important to the individual and group when making decisions about their experience of a destination, whether to visit again, and their feelings in relation to the tourist experience of place». L'image finaleva donc participer au processus de construction territoriale.

Le principal problème que rencontre le touriste dans le choix de sa destination est que l'image qu'il se fait du territoire (la «pré-image», celle qui est antérieure au déplacement) reste impalpable et ne se concrétisera qu'une fois le voyage effectué. S'il se rend sur le territoire pour la première fois, le touriste peut percevoir l'achat de la prestation comme risquée. Le risque est d'autant plus ressenti que les produits touristiques «représentent souvent un investissement financier et temporel important, en d'autres termes des produits pour lesquels l'implication est forte» (Frochot etLegohérel, 2007 : 45). Par ailleurs, l'intangibilité et l'hétérogénéité des services ont aussi pour effet d'augmenter le caractère abstrait de leur contenu et le risque perçu (De Ruyter etal., 2001). Le consommateur touristique va donc essayer de trouver des éléments rassurants ou qui renvoient une image rassurante. Comme évoqué en introduction, MacCannell(1989:41) démontre qu'une attraction touristique est "an empiricalrelationshipbetweenatourist, a sight and a marker». Leiper(1990: 381) reprend cette idée en précisant que le touriste est confronté aux marqueurs tout au long de son séjour :avant son départ (générating marker), pendant son déplacement (transit marker) et finalement in 


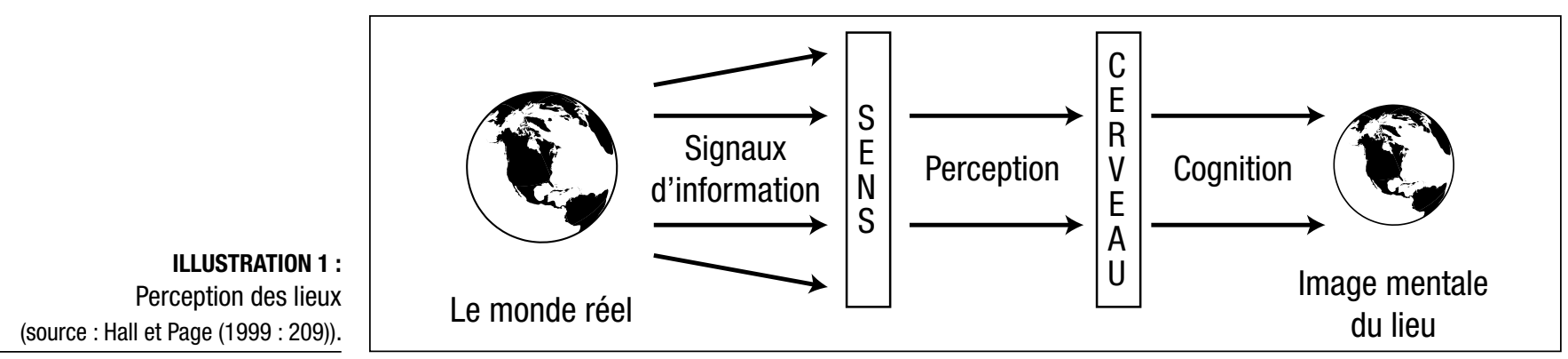

situ(continuous marker).Il privilégie les témoignages de qualité et,parmi eux, nous le supposons, les labels touristiques qui participent à la construction de l'image territoriale.

Les travaux sur les labels sont encore peu nombreux et à fortiori ceuxsur les labels touristiques. Le dictionnaire Larousse (2010 : 678) définit le label comme un «signe garantissant la qualité de quelque chose». C'est une définition relativement large qui permet d'intégrer tout et n'importe quoi. Le code de la consommation précise :

[Il] constitue une certification de produit ou de service soumise aux dispositions de la présente section l'activité par laquelle un organisme, distinct du fabricant, de l'importateur, du vendeur, du prestataire ou du client, atteste qu'un produit, un service ou une combinaison de produits et de services est conforme à des caractéristiques décrites dans un référentiel de certification (PICOD, art. L.115-27).

Les travaux de Larceneux(2003a, 2003b)concluent qu'un label est «un signe qui informe sur les dimensions spécifiques de la qualité, sous quelque forme que ce se soit, d'un produit ou service qui émane d'un organisme différent de l'entreprise propriétaire des biens ou services» (Larceneux, 2003b). Cette définition est retenue,car elle évoqueà notre avis les deux dimensions fondamentales d'un label :

- Le témoignage de la qualité du produit ou du service;

- L'attribution du label par un organisme différent du producteur.

Pendant longtemps les labels ont été l'apanage des entreprises privées, mais, depuis quelques années, ils sont utilisés par des organismes publics. Confrontés au besoin de rentabiliser les investissements et au besoin de protéger le patrimoine(Bourdeau et Marcotte, 2006), certains organismes publics ont eu recours à des stratégies de labellisation. En matière de tourisme, la concurrence internationale pousse à l'utilisation de stratégies marketing plus professionnelles pour se différencier. Le coût des investissements touristiques étant important, les territoires visent désormais la rentabilité. En garantissant une certaine qualité, les labels touristiques peuvent contribuer à l'augmentation de la fréquentation touristique et donc à une meilleure rentabilité des équipements. L'augmentation du nombre d'entrées dans un site culturel vient également renforcer un budget en diminution constante du fait de la réduction des subventions publiques.

Dans le monde touristique, il existe plusieurs labels reconnus propres à chaque domaine de ce vaste secteur économique. On citera par exemple le guide Michelin et ses étoiles pour la restauration, le label UNESCO pour le patrimoine culturel et naturel, les recommandations de différents guides pour l'hébergement, etc. Ce sont essentiellement des labels expérientiels qui transmettent une information subjective émanant d'experts sur la qualité de l'expérience de consommation (Larceneux, 2003a). Avec la diffusion d'Internet, les experts sont plus nombreux et les blogues sont autant d'avis de touristes ayant essayé un service et qui le recommandent ou non. On peut aussi parler de «label récompense» (Chameroy et Chandon,2010) qui est émis par des professionnels du secteur, cautionnés par les pouvoirs publics. Ce n'est pas nécessairement une garantie, mais ce dernier type de labelinforme le consommateur de jugements d'autrui sur le produit.

Les labels sont donc faits,entre autres, pour rassurer les touristes et les futurs consommateurs sur la qualité du produit ou du service. Toutefois, interviennent-ils réellement dans la construction de l'image territoriale et donc dans le choix d'une destination puisque celle-ci est fondamentale?

\section{Rôle du label UNESCO dans le choix d'une destination}

Certaines études ont été menées sur le sujet et notamment celle d'Isabelle Prim-Allazet al. (2008). Après un travail d'enquête réalisé auprès de 500 personnes réparties dans plusieurs pays du monde, ils aboutissent à la conclusion que les labels touristiques ne sont pas pris en compte dans le choix d'une destination mais qu'ils interviennent une fois que celle-ci est choisie. Cependant,de notre point de vue, et les auteurs en sont conscients, ce travail présente une limite : l'échantillon est essentiellement constitué d'un public jeune et de formation supérieure (ceci tient essentiellement à la méthodologie d'enquête retenue) et n'est donc pas représentatif de l'ensemble de la clientèle touristique.

\section{Les aspects méthodologiques}

Le 16 novembre 1972 constatant que «le patrimoine culturel et le patrimoine naturel sont de plus en plus menacés» et que «la protection de ce patrimoine à l'échelon national reste souvent incomplète » (préambule de la convention de l'UNESCO, 1972), l'UNESCO adopte une convention concernant la protection du patrimoine mondial, culturel et naturel. Les états signataires reconnaissent que certains biens patrimoniaux ont une valeur exceptionnelle qui nécessite leur préservation en tant que patrimoine mondial de l'humanité. Il est donc instauré une liste du patrimoine mondial qui recense ces richesses méritant une protection internationale (art. 11-2). Au-delà de la seule 
protection du patrimoine, l'UNESCO pousse aussi les États à valoriser leurs richesses (art. 4-2). Le processus d'attribution du label est long et technique. Les États déposent chaque année un inventaire des biens qu' ils souhaitent inscrire sur la liste du patrimoine mondial. Chaque dossier doit comporter des informations techniques sur le bien pour être ensuite étudié par des experts compétents dans ce domaine, membres de l'ICOMOS - c'est pourquoi on peut parler de label expérientiel. À l'origine, le label UNESCO est un label technique visant à identifier les sites patrimoniaux menacés ou ayant une valeur universelle. Toutefois, la charte insiste fortement sur la «mise en valeur» du patrimoine (art. 5) et demande aux États «d'adopter une politique générale visant à assigner une fonction au patrimoine culturel et naturel dans la vie collective» (art. 5-1). Or, il apparaît que généralement les sites UNESCO sont utilisés à des fins touristiques, et la plupart sont ouverts à la visite. Finalement, le label UNESCO est devenu au fil du temps un label touristique, un gage de qualité pour les touristes. Visiter un site UNESCO, c'est visiter un site unique! Les experts de l'ICOMOS peuvent alors être comparés à des critiques touristiques : leur jugement certifie de l'intérêt du lieu. C'est en cela que l'on peut parler de label expérientiel, même s'il garde une valeur scientifique importante puisque s'appuyant sur des critères précis d'évaluation. Contrairement aux étoiles du Michelin ou celles des hôtels, le label UNESCO ne garantit pas un service de qualité, mais garantit un site d'intérêt historique ou esthétique. La mise en valeur dépendra toujours du propriétaire et elle peut être bonne ou mauvaise. La dimension expérientielle s'arrête aux caractéristiques intrinsèques du site et ne prend pas en compte sa dimension touristique, ce que les visiteurs oublient souvent. C'est après avis de ces experts que l'UNESCO décide ou non d'attribuer le label aux biens proposés. Dès lors l'organisme propriétaire peut l'utiliser dans sa communication. En octobre 2011, 936 biens culturels, naturels et mixtes étaient inscrits sur la liste.

La France fait partie des États qui possèdent le plus de sites inscrits sur la liste du patrimoine mondial. Avec l'inscription des Causses et des Cévennes, et des sites palafittiques préhistoriques des Alpes en 2011, ce sont 37 sites qui bénéficient du label. De nombreux autres sites ont essayé d'intégrer la liste dont le parc de la Vanoise, le phare de Cordouan ou encore le château de Vaux-le-Vicomte (36 sites au total selon le site Internet de l'UNESCO).Il y a donc une vraie concurrence entre les territoires pour obtenir ce label prestigieux qui donne une visibilité mondiale aux biens inscrits. Il semblerait en effet que son obtention entraîne une augmentation de $20 \%$ de la fréquentation touristique (c'est du moins le chiffre annoncé par certains journaux comme La dépêche, Le Figaro ou Le Monde), mais ces données mériteraient d'être affinées. Toujours est-il qu'il existe un véritable intérêt des acteurs publics et privés français pour obtenir l'inscription sur la liste du patrimoine mondial.Nous tentons ici de montrer l'intérêt de la démarche.

Afin de mesurer l'impact du label UNESCO dans le processus de choix d'une destination touristique, nous avons effectué une enquête dans trois sites UNESCO : Besançon (la citadelle), Provins (la cité médiévale) et Avignon (le palais des papes et le pont Saint-Bénezet). Ce sont trois sites à la réputation touristique différente : Avignon est sûrement le plus connu des trois.
Les enquêtes ont été réalisées dans le cadre d'un mémoire de recherche en tourisme encore en cours de finalisation à l'heure actuelle. Les questionnaires ont été soumis à des touristes français et internationaux (après avoir été traduits en anglais). Dans tous les cas, ils ont été administrés en face à face au cours de l'automne 2010 à proximité des sites UNESCO. Les touristes sont moins nombreux à cette période de l'année, mais la réalisation des enquêtes la fin de semaine et lors des vacances de la Toussaint a permis d'obtenir des résultats satisfaisants en termes de nombre de questionnaires.

Sur chacun des trois sites, 150 questionnaires ont été administrés. Seuls 424 se sont avérés utilisables, car 26 présentaient des incohérences ou n'étaient remplis que partiellement. Par facilité, nous avons opté pour un échantillon non probabiliste dans la mesure où nous ne disposions pas d'une population de référence. Les touristes ont été interrogés au hasard des rencontres, mais toujours à proximité du site UNESCO.On peut donc considérer qu'il s'agit d'un échantillon à l'aveuglette avec tous les inconvénients que cela comporte. C'est pourquoi l'enquête est ici considérée comme exploratoire. Seuls des touristes et des excursionnistes ont été interrogés, c'est-à-dire des personnes habitants hors du bassin de vie concerné et venus dans le but de découvrir la ville où est localisé le site UNESCO ou le site UNESCO lui-même. Nous pouvons considérer que nous avons une bonne répartition sociodémographique de notre échantillon : $57 \%$ de femmes (souvent plus enclines à répondre que les hommes), $23 \%$ de moins de 25 ans, $45 \%$ de 25 à 60 ans et $32 \%$ de plus de 60 ans. La surreprésentation des personnes âgées peut s'expliquer par la période d'étude : ils sont plus nombreux à partir horssaison. Enfin nous avons $87 \%$ de Français et $13 \%$ d'internationaux, ceux-ci ont surtout été rencontrés en Avignon. Les questionnaires ont tous été traités avec le logiciel Sphinx.

Par ces questionnaires nous avons cherché à répondre à deux interrogations :

- Le label UNESCO est-il connu des touristes?

- Le label UNESCO a-t-il un impact dans le choix d'une destination touristique?

Par souci de concision, les questions seront présentées au fur et à mesure de l'analyse des résultats.

\section{La connaissance du label UNESCO}

Lorsque l'on demande aux visiteurs s'ils connaissent la liste du patrimoine mondial, ils sont près de $90 \%$ à savoir qu'elle existe. En revanche, ils ont beaucoup plus de difficultés à expliquer en quoi consiste cette liste. La réponse la plus fréquemment donnée est «la liste des monuments les plus connus» (60\% en moyenne). Pour beaucoup, il s'agit d'un inventaire sans autre fonction. Ils sont presque $28 \%$ à considérer qu'elle recense le patrimoine menacé, et pour $20 \%$, c'est la liste des sites à protéger.On peut donc considérer qu'un touriste sur cinq connaît l'intérêt d'inscrire un bien sur la liste du patrimoine mondial. Notons cependant que le volet valorisation n’a jamais été évoqué. Dans la grande diversité des réponses, puisque la question était ouverte, il apparait que le patrimoine naturel n'est que rarement cité : peut-être parce que l'on porte davantage d'intérêt aux richesses bâties dans les sociétés occidentales et que le terme "patrimoine» est souvent assimilé à 
«bâtiment» (Audrerie et al., 1998). Il faut donc retenir qu'il y a un vrai travail d'éducation et d'information à mener sur ce label qui est pourtant connu.

Par ailleurs, il a été demandé aux touristes de citer spontanément cinq sites français qui selon eux bénéficient du label UNESCO. Cinq types de réponses sont apparus :

- Les touristes citent d'abord les sites les plus prestigieux et les plus connus du patrimoine français. Ainsi le château de Versailles et le Mont Saint-Michel sont donnés par plus de $60 \%$ des répondants. D’autres sont donnés à titre individuel alors qu'ils appartiennent à un ensemble (la Tour Eiffel, le château de Chambord, Notre-Dame-de-Paris, etc.). Il semblerait que ces monuments soient cités parce que les répondants estiment qu'ils «méritent» ce label mais, pour beaucoup, ils ne sont pas sûr qu'ils le possèdent réellement;

- Nous constatons également ce qu'on peut appeler un "effet proximité». En effet, les personnes interrogées sont capables de citer des éléments du patrimoine labellisés UNESCO qui se trouvent à proximité de leur lieu de résidence (cathédrale d'Amiens pour les Picards, Strasbourg pour les Alsaciens, etc.) ou de l'endroit visité (Orange et Arles pour les touristes à Avignon, par exemple). Cela témoigne d'un intérêt pour le label, qu'ils soient chez eux ou en vacances;

-Un « effet visite» doit être relevé. Les personnes interrogées ont cité les sites qu'elles avaient déjà visités et notamment ceux qu'elles étaient en train de découvrir. Ainsi Provins, Avignon et Besançon ont été cités par plus de $75 \%$ des touristes, obtenant la tête du classement;

- Un ensemble de sites n'a jamais été cité : le golfe de Porto, l'abbaye de Fontenay, la saline d'Arc-et-Senans, la vallée de la Vézère, les chemins de Saint-Jacques-de-Compostelle ou encore le Havre. Cela tient à leur manque de notoriété, mais aussi au fait qu'aucun touriste originaire de ces régions n'a été interrogé : nous n'avons par exemple pas croisé de Corses;

-Enfin, il y a ce que l'on peut appeler un «effet zoom». Les sites qui ont été inscrits en 2010 (Albi et la Réunion) sont évoqués par plus de $10 \%$ des répondants. Ceci paraît important au regard de leur notoriété touristique. Il y a fort à penser que, pour une même enquête effectuée l'année prochaine, ils n'apparaissent que très peu.

On peut aussi retenir qu'il n'y a quasiment pas eu d'erreur dans les réponses données. Tous les sites évoqués sont inscrits à l'UNESCO (à l'exception de Marseille, cité 14 fois, oude Toulouse, cité six fois).

Ces conclusions sont confirmées par la question suivante :«Parmi ces sites, quels sont ceux qui, d'après vous, sont inscrits sur la liste du patrimoine mondial?» La liste des 35 sites français inscrits au patrimoine mondial de l'humanité en 2010 a été donnée à chaque personne,et nous leur avons demandé quels étaient les sites qu'ils connaissaient et quels étaient ceux qui étaient inscrits UNESCO, d'après eux. On constate que(voir tableau 1) :

- les sites les plus connus et les plus médiatisés sont largement relevés : Versailles, Paris, Carcassonne, le Mont SaintMichel, le Val de Loire, etc.;
- les sites les moins connus sont oubliés : Fontenay, Vézelay, Porto, Gavarnie, les beffrois du nord, etc.;

- l'effet proximité est présent : on cite les bâtiments des régions dont on est originaire ou qu'on a déjà visités.

Enfin, pour terminer cette partie, il a été demandé aux touristes combien de sites français étaient inscrits sur la liste du patrimoine mondial. Pour près de $40 \%$ d'entre eux, il y en a entre 20 et 30 , soit moins que la réalité, et pour plus de $61 \%$, moins de 30 . Il y a donc là un effort de communication à effectuer pour informer sur le label UNESCO.

\section{Le label UNESCO comme facteur de choix de destination touristique}

Après avoir vérifié que le label était connu des touristes, nous avons voulu savoir si celui-ci était déclencheur de voyage et si le site était la première motivation du déplacement.

Lorsque l'on demande aux touristes s'ils savaient avant de partir que le site était labellisé UNESCO, il apparaît deux tendances :

- Pour Avignon, site connu nationalement (voir mondialement) de par le rôle qu'elle a joué dans l'histoire de la papauté, seul $52 \%$ des touristes savaient qu'elle était inscrite sur la liste du patrimoine mondial. Les autres l'ignoraient et l'ont appris sur place (et certains l'ignorent encore).

- En revanche à Besançon et Provins, plus de $65 \%$ des touristes français et $80 \%$ des touristes étrangers le savaient avant de venir. Ceci témoigne d'une prise d'information avant de venir.

On a constaté par ailleurs que, pour Besançon et Provins, l'absence de label n'aurait peut-être pas $(45 \%)$ ou pas $(21 \%)$ provoqué de déplacement. L'inscription sur la liste du patrimoine mondial a donc été déclencheur de voyage. Ceci est confirmé par une autre question, plus directe : «Êtes-vous venu parce que le site était inscrit sur la liste du patrimoine mondial?». Pour Besançon et Provins, c'est une justification importante : près de $70 \%$ dans les deux cas. Pour Avignon, le label joue un rôle moindre (48\%). Les autres motivations qui sont évoquées sont la réputation, la proximité, la curiosité, l'étape... Il faut donc retenir que, pour des sites «connus» tels Avignon, Versailles ou le Mont Saint-Michel, le label UNESCO joue un rôle important mais pas essentiel dans le choix de la destination. En revanche, il est fondamental pour des sites de «second rang» tels Provins, Saint-Savin-sur-Gartempe, Fontenay ou Albi. Ainsi un couple nous a déclaré : «Nous n'étions jamais venu dans le Doubs et nous ne savions pas quel site visiter, alors nous avons choisi un site reconnu mondialement comme Besançon.» Le label UNESCO aide donc aussi lorsque la destination est choisie comme le montrait l'étude d'Isabelle PrimAllazet al. (2008). Néanmoins, l'enquête montre qu'il est aussi un facteur de choix, surtout pour les sites peu réputés. Comme eux, nous constatons que, chez les jeunes (moins de 25 ans), il a un impact moindre (60\% pour Besançon et Provins, $34 \%$ pour Avignon), mais il est très élevé chez les personnes âgées (plus de $82 \%$ à Besançon et Provins) et les touristes étrangers (86\%). Précisons que, en plus de la méthodologie différente et du panel choisi, l'enquête ne portait que sur le label UNESCO et a été réalisée dans des sites UNESCO. Ceci pèse sans aucun doute sur les réponses obtenues. 
Tableau 1: Question posée aux participants à l'étude : “Connaissez-vous les sites suivants? Sont-ils selon vous inscrits sur la liste du patrimoine mondial de l'UNESCO ? " (enquête, 2010, en pourcentage)

\begin{tabular}{|c|c|c|c|c|c|}
\hline Sites & Je connais & Je ne connais pas & Est inscrit selon moi & $\begin{array}{l}\text { N'est pas inscrit } \\
\text { selon moi }\end{array}$ & Ne sais pas \\
\hline Mont St-Michel & 100 & 0 & 97 & 3 & 0 \\
\hline Quais de Paris & 100 & 0 & 95 & 2 & 3 \\
\hline Château de Versailles & 98 & 2 & 93 & 4 & 3 \\
\hline Bordeaux & 93 & 7 & 43 & 36 & 21 \\
\hline Pont du Gard & 89 & 11 & 79 & 14 & 7 \\
\hline Carcassonne & 89 & 11 & 89 & 10 & 1 \\
\hline Lyon & 87 & 13 & 55 & 21 & 24 \\
\hline Château de Fontainebleau & 86 & 14 & 85 & 12 & 3 \\
\hline Strasbourg & 86 & 14 & 31 & 41 & 28 \\
\hline Châteaux de la Loire & 85 & 15 & 86 & 5 & 9 \\
\hline Places de Nancy & 71 & 29 & 52 & 26 & 22 \\
\hline Cité médiévale de Provins & 68 & 32 & 68 & 21 & 11 \\
\hline Palais des papes d'Avignon & 65 & 35 & 64 & 22 & 14 \\
\hline Cathédrale de Reims & 64 & 36 & 75 & 13 & 12 \\
\hline Cathédrale de Chartres & 57 & 43 & 64 & 18 & 18 \\
\hline Île de la Réunion & 56 & 44 & 53 & 35 & 12 \\
\hline Cathédrale d'Amiens & 51 & 49 & 41 & 36 & 23 \\
\hline Fortifications de Vauban & 47 & 53 & 41 & 25 & 24 \\
\hline Saint-Émilion & 43 & 57 & 21 & 49 & 30 \\
\hline Grottes préhistoriques de Dordogne & 41 & 59 & 76 & 21 & 3 \\
\hline Cathédrale de Bourges & 36 & 64 & 39 & 40 & 21 \\
\hline Saline d'Arc-et-Senans & 32 & 68 & 34 & 31 & 35 \\
\hline Arles & 31 & 69 & 26 & 43 & 31 \\
\hline Lagons de Nouvelle-Calédonie & 31 & 69 & 57 & 29 & 13 \\
\hline Le Havre & 27 & 73 & 15 & 67 & 18 \\
\hline Canal du Midi & 26 & 74 & 23 & 41 & 26 \\
\hline Théâtre antique d'Orange & 25 & 75 & 24 & 57 & 19 \\
\hline Chemins de St-Jacques & 23 & 77 & 35 & 41 & 24 \\
\hline Albi & 21 & 79 & 58 & 16 & 26 \\
\hline Cirque de Gavarnie & 21 & 79 & 35 & 30 & 35 \\
\hline Beffrois du NPC & 14 & 86 & 26 & 52 & 22 \\
\hline Abbaye de Fontenay & 12 & 88 & 28 & 43 & 29 \\
\hline Golfe de Porto & 11 & 89 & 12 & 46 & 42 \\
\hline Basilique de Vézelay & 9 & 91 & 16 & 58 & 26 \\
\hline Abbaye de St-Savin-sur-Gartempe & 4 & 96 & 12 & 63 & 25 \\
\hline
\end{tabular}

Pourquoi ce label est-il un critère de choix? Quelle image véhicule-t-il? À ces questions, les personnes interrogées mettent clairement en avant la qualité du site. Pour beaucoup (71\%), la reconnaissance internationale est associée à un gage de qualité, sans préciser par ailleurs ce qu'ils mettent derrière ce mot (qualité de conservation, qualité architecturale, qualité de l'accueil?). Deux autres termes apparaissent avec récurrence : "prestige» et "exceptionnalité». On comprend plus aisément la logique de ces deux termes : la reconnaissance mondiale suppose en effet que le site est exceptionnel puisqu'il fait partie des rares à être inscrits sur la liste et prestigieux dans la mesure où il figure aux côté de sites aussi célèbres que 
les pyramides d'Égypte ou la grande muraille de Chine. Ces adjectifs positifs donnent donc une pré-image valorisante du territoire qui possède le label UNESCO.

Enfin, lorsque l'on interroge les touristes sur le vecteur de connaissance du label, il ressort qu'Internet (43\%) et le bouche-à-oreille (39\%) sont les principales sources d'information.

Cette étude de terrain met donc en avant deux éléments importants :

- Les touristes connaissent la liste du patrimoine mondial, mais ils ne savent pas vraiment quelle est sa fonction. Ils lui associent des qualités positives mais ont des difficultés à identifier les sites français inscrits sur cette liste. Seuls les sites les plus connus sont cités. Il y a donc incontestablement un travail d'information et de communication à effectuer sur ce label;

- Le label UNESCO est un élément essentiel dans le choix d'une destination, notamment pour les étrangers. Il doit donc être mis en avant dans la communication touristique pour donner une image positive du territoire et renforcer son attractivité. Il doit être utilisé comme les marqueurs évoqués par Leiper (1990) et être utilisé dans la communication avant, pendant et après le déplacement du touriste.

$\mathrm{Au}$ terme de cette analyse, et considérant que le fait de posséder un site inscrit sur la liste du patrimoine mondial n'est pas anodin pour un territoire, nous avons voulu savoir comment les acteurs locaux utilisaient le label UNESCO dans leur communication.

\section{Utilisation du label UNESCO dans la communication touristique}

La communication touristique joue un rôle important dans la construction de la pré-image des territoires et donc dans le choix d'une destination touristique. On considère souvent que le bouche-à-oreille est le premier vecteur de communication dans le domaine touristique. Comme le fait remarquer Pierre Frustier (1999: 32), "l'oreille est déjà sensibilisée au site» par l'ensemble du discours médiatique. La communication permet d'associer des éléments qui finissent par composer une image dans le subconscient de l'individu. Qu'on le veuille ou non, la communication touristique va donc influer sur la construction de l'image territoriale. Étant donné que le label UNESCO donne une image positive et rassurante, on peut supposer qu'il est utilisé abondamment par les acteurs dont les sites bénéficient de cette appellation. C'est donc ce que nous avons voulu savoir.

En matière de communication touristique, il existe plusieurs médias possibles : la presse, la télévision, la radio, l'affichage extérieur, le cinéma, Internet et les brochures touristiques. Tous ont leurs avantages et leurs défauts, et sont utilisés par les acteurs territoriaux pour vanter leur destination. Depuis quelques années, Internet est devenu un des principaux médias de communication touristique. En effet, face à l'explosion du nombre d'internautes, les comités régionaux du tourisme (CRT), les comités départementaux du tourisme (CDT) ou les offices de tourisme ont développé des sites Web de plus en plus attractifs. Près de $50 \%$ des ménages français et européens ont une connexion Internet. Le nombre de visiteurs potentiels est donc considérable. Par ailleurs, il apparaît que le secteur de l'e-tourisme représenterait à lui seul $46 \%$ du volume financier du e-commerce en France (Raffour, 2007). Les sites Internet seraient en passe de supplanter les agences de voyages dans la vente de produits touristiques. Une étude d'opinionsen juin 2005 (dans Victor, 2007) montre que, pour préparer leurs vacances, $20 \%$ des Français fréquentent les sites des offices de tourisme et autres sites institutionnels. Avec l'apparition des téléphones intelligents, le touriste reste connecté durant ses vacances et peut rechercher des informations sur Internet sans se rendre dans les offices de tourisme. Il semble donc que le Web devienne bien le principal vecteur d'informations. C'est donc sur ce média que les recherches ont été centrées.

Dans le but de mesurer comment était utilisé le label UNESCO dans la communication touristique, les sites Internet des 35 sites inscrits sur la liste du patrimoine mondial ont été analysés. Ce travail a rencontré quelques difficultés :

- pour certains biens, il n'existe pas de site Internet spécifique (places de Nancy, cité épiscopale d'Albi, site historique de Lyon, cathédrale d'Amiens, etc.);

- pour d'autres, il existe plusieurs sites, car les biens sont éclatés dans l'espace : c'est le cas pour les ensembles (chemins de Saint-Jacques-de-Compostelle, fortifications de Vauban, beffrois du Nord-Pas de Calais, etc.). Pour ceuxlà, il existe en plus un site spécifique, mais que peu de touristes seraient susceptibles de consulter en premier à notre avis;

- pour deux biens, il n'existe aucun site : le canal du Midi et les lagons de Nouvelle-Calédonie.

Nous avons donc dû nous résoudre à des choix. Pour les sites monumentaux isolés, nous avons étudié le site Internet du gestionnaire (ex : château de Versailles, abbaye de Fontenay, saline d'Arc-et-Senans). Pour les quartiers urbains inscrits, nous avons étudié le site de l'office de tourisme de la ville en question (Bordeaux, Albi, Provins, etc.). Pour les monuments isolés mais qui ne disposent pas de sites spécifiques, comme c'est le cas pour les cathédrales, nous avons choisi de retenir le site Web des offices de tourisme. Enfin pour les ensembles (Saint-Jacques-de-Compostelle, fortifications de Vauban, etc.), nous nous sommes penchés sur le site dédié. Toutefois, ceci présente une limite car, ces sites étant dédiés aux biens labellisés UNESCO, les références à ce label sont très nombreuses. On peut donc considérer qu'ils ne sont pas représentatifs. Il est par ailleurs peu probable qu'un touriste consulte ce type de site pour choisir sa destination de vacances. Ils seront donc laissés de côté pour l'analyse finale.

Dans un premier temps, nous avons observé sept critères :

- la mention du label UNESCO sur la page de recherche Google, moteur de recherche le plus utilisé;

-l'apparition du logo UNESCO sur la page d'accueil du site;

- l'utilisation du label dans l'appellation du site (ex. : Carcassonne, patrimoine mondial de l'humanité)

- l'évocation du label sur la page d'accueil;

- la visibilité du label sur la page d'accueil;

- l'existence d'une page dédiée au label UNESCO;

- la référence au label dans le texte du site Internet. 
Pour chacun de ces sept critères une note entre 0 et 5 a été attribuée : 0 lorsque le critère n'est pas présent, 5 lorsqu'il l'est. Seul le critère «visibilité» a fait l'objet d'une gradation de 0 à 5 puisque c'est le seul pour lequel il existe des niveaux (il est possible d'établir une échelle précise de visibilité du logo sur un site).Dans un second temps, une moyenne a été effectuée, qui donne un classement des sites Internet en fonction de l'importance donné au label UNESCO. Seuls les sites Web des ensembles inscrits n'ont pas été évalués pour les raisons évoquées précédemment.

Les résultats de cette évaluation (voir tableau 2) laissent apparaître plusieurs éléments intéressants. On constate tout d'abord que les sites Internet qui mettent le plus en avant le label UNESCO sont avant tout ceux des biens les moins «connus» sur le plan touristique, tel l'abbaye de Fontenay, l'abbatiale de Saint-Savin-sur-Gartempe ou encore Provins. Pour ce dernier, M. Robin, le directeur de l'office de tourisme, nous a clairement laissé entendre que le label UNESCO était un élément fondamental pour attirer les touristes et qu'il était «quasiment vital» de communiquer dessus (communication personnelle, 2010). Tout laisse à penser qu'il en est de même pour les autres biens évoqués. L'autre grand utilisateur du label est l'Office de tourisme du Havre, car il permet de changer l'image de la ville : passer de l'image de port industriel à celle de destination touristique patrimoniale. Ceci est cohérent avec l'enquête de terrain. Les sites les moins connus doivent communiquer beaucoup pour attirer les touristes. Certains l'ont bien compris (Saint-Savin-sur-Gartempe, Provins) mais d'autres doivent encore s'améliorer : Vézelay n'est connu que par $9 \%$ des personnes interrogées et met assez peu en avant son label UNESCO.

En revanche les sites les plus connus utilisent très peu le label UNESCO dans leur communication. Ainsi sur le site Internet de Paris et du château de Fontainebleau, on ne trouve aucune référence à l'UNESCO. Il en est de même pour le Mont Saint-Michel et le château de Versailles. On peut considérer qu'ils n'ont pas besoin de l'utiliser, leur seul nom suffisant pour attirer les touristes. Les biens localisés dans les grandes régions touristiques utilisent eux aussi assez peu le label dans leur communication Internet : ils peuvent attirer des visiteurs sans ce label. Albi et la Réunion, inscrits cette année, évoquent assez peu leur inscription; il faudra voir l'évolution dans les années à venir. Enfin, des sites UNESCO mais localisés dans des régions peu touristiques (Chartres, Amiens, Vézelay, etc.) sont également peu diserts sur le label dans leur promotion; pourtant au vue des résultats de l'enquête une meilleure communication pourrait leur être bénéfique.

Il faut noter deux exceptions à ce schéma. Carcassonne et le pont du Gard, pourtant mondialement connus, usent beaucoup du label dans leur communication allant jusqu'à l'utiliser dans leur appellation : «Carcassonne, patrimoine mondial» et «le pont du Gard, haut-lieu du patrimoine mondial».

Pour compléter cette analyse, nous avons également consulté les sites Internet des communes, des comités départementaux de tourisme (CDT) et des comités régionaux de tourisme (CRT). Nous nous sommes contentés d'observer la place qui était donné au label UNESCO dans leur communication. Là encore, on peut noter que :

- en dehors de l'Île de France, tous les CRT communiquent sur leurs sites UNESCO. Cependant, certains comme la Bourgogne, l'Aquitaine ou le Languedoc-Roussillon leur réservent une page spéciale. Au total, la moitié des CRT agissent ainsi pour mettre en avant le patrimoine mondial. - pour les CDT, on peut dire la même chose : certains se contentent d'en parler, d'autres leur accordent une place importante (Gironde, Tarn, Rhône, Eure et Loir, Yonne...). Il faut aussi noter que certains n'en parlent pas du tout (Gard, Bas-Rhin, Seine-Maritime...).

Tableau 2: Note obtenue pour la communication des différents sites

\begin{tabular}{|c|c|c|c|}
\hline Sites & Notes & Sites & Notes \\
\hline Palais et parc de Fontainebleau & 0 & Cathédrale de Chartres & 1,71 \\
\hline Paris, rives de la Seine & 0 & Basilique et colline de Vézelay & 1,85 \\
\hline Mont Saint-Michel et sa baie & 0,71 & Saline d'Arc-et-Senans & 2,42 \\
\hline Palais des papes et pont d'Avignon & 0,71 & Golfe de Porto & 2,42 \\
\hline Places de Nancy & 0,71 & Juridiction de Saint-Émilion & 2,78 \\
\hline Cathédrale d'Amiens & 0,71 & Strasbourg - Grande Île & 2,85 \\
\hline Cathédrale de Bourges & 0,71 & Ville fortifiée de Carcassonne & 3,14 \\
\hline Reims & 1 & Gavarnie - Mont Perdu & 3,14 \\
\hline Palais et parc de Versailles & 1,42 & Abbaye de Fontenay & 3,28 \\
\hline Site historique de Lyon & 1,42 & Pont du Gard & 3,28 \\
\hline Pitons, cirques et remparts de la Réunion & 1,42 & Abbatiale de Saint-Savin-sur-Gartempe & 3,42 \\
\hline Théâtre antique d'Orange & 1,71 & Le Havre, ville reconstruite par A. Perret & 3,57 \\
\hline Arles, monuments romains et romans & 1,71 & Provins, villes de foires médiévales & 4,85 \\
\hline La cité épiscopale d'Albi & 1,71 & Bordeaux, port de la Lune & 5 \\
\hline
\end{tabular}

Source : compilation de l'auteur. 
- il en va de même pour les communes : Orange, Strasbourg, Bordeaux, Arles, Lyon ou Le Havre communiquent beaucoup sur leur label. Saint-Émilion a même créé son site municipal autour du label.

L'attitude de certaines communes et de certains CRT et CDT montre que beaucoup d'entre eux ont mesuré l'intérêt de mettre en avant le label UNESCO dans leur communication touristique. Réserver une page spéciale aux sites UNESCO prouve qu'ils considèrent qu'ils sont un facteur d'attractivité et qu'ils ont un impact dans le choix d'une destination touristique. Il s'agit alors d'être le plus original possible pour parvenir à se démarquer des autres.C'est ce que tente de faire Saint-Émilion.

$\mathrm{Au}$ terme de ce travail d'observation, on peut considérer que l'utilisation du label UNESCO dans la communication touristique sur Internet est très différente d'un site à l'autre. Il est difficile d'établir une typologie précise, même s'il semble que les sites qui utilisent davantage le label soient ceux qui en ont le plus besoin. En effet, comme nous l'avons noté, les sites à priori peu connus touristiquement font une large place au label UNESCO sur leur site Internet. Lorsque l'on croise la fréquentation touristique et la note obtenue pour la communication (voir illustration 2), on constate une corrélation inverse : plus le bien est fréquenté, moins il communique sur le label UNESCO. Ainsi comme nous l'évoquions précédemment, Versailles, le Mont Saint-Michel et Lyon utilisent peu leur logo mais reçoivent plus de 3 millions de touristes. À l'opposé, les "petits» sites comme Fontenay, Saint-Savinsur-Gartempe ou Arc-et-Senans communiquent fortement sur leur label. Il y a évidemment quelques exceptions : le château de Fontainebleau qui reçoit moins de 400000 visiteurs ne fait pas du tout de promotion sur le fait qu'il soit inscrit sur la liste du patrimoine mondial. À l'inverse, malgré sa fréquentation, Carcassonne continue d'user du label UNESCO. Stratégiquement, c'est sans doute payant.

L'inscription sur la liste du patrimoine mondial influence positivement le choix d'une destination. Elle est un outil utile pour distinguer deux destinations. Pour beaucoup, elle est un gage de qualité et rassure. Nous pensons donc que la communication sur le label UNESCO a un impact sur la fréquentation. Provins en est un bon exemple : avec seulement 26000 habitants, la cité médiévale attire 800000 touristes

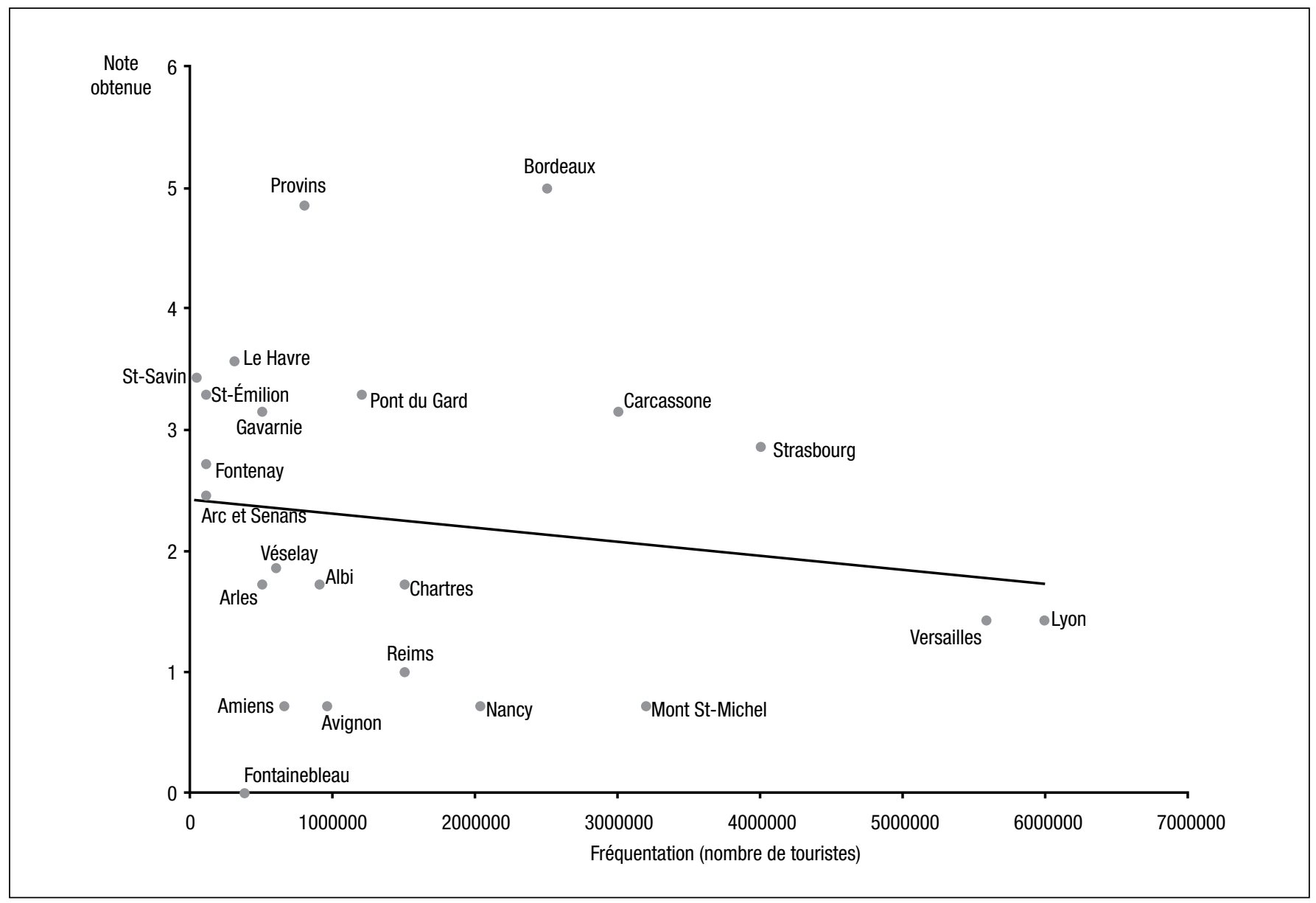

ILLUSTRATION 2 : Communication et fréquentation des sites UNESCO (enquête, 2010) (source : données compilées par l'auteur à partir de l'enquête et des chiffres fournis par les offices de tourisme et les CDT). 
chaque année alors que dans l'opinion elle est peu connue (seul $26 \%$ des touristes rencontrés à Besançon et Avignon la connaissent, notons qu'il s'agit surtout d'habitants de l'Île-deFrance). À l'inverse, Fontainebleau, très connu dans l'opinion (86\% affirme connaître le château) ne reçoit que 400000 visiteurs. Ces deux sites sont emblématiques dans la mesure où ils sont tous les deux très proches de l'agglomération parisienne et susceptibles d'attirer une clientèle identique.Pourtant, c'est le moins connu des deux qui attire le plus. On peut donc considérer que, pour les destinations à faible notoriété, l'usage du label UNESCO dans la communication est une contrainte : sans lui, la fréquentation reste faible (on peut comparer l'abbaye de Fontenay et la cité de Provins). À l'opposé, pour les sites qui disposent d'une grande notoriété, l'utilisation du label UNESCO dans la communication est un outil complémentaire mais pas indispensable : Versailles comme le Mont Saint-Michel s'en passent aisément, alors que Carcassonne le met fortement en avant. Il faut noter que les sites connus qui l'utilisent peu, tel Fontainebleau, restent «peu» fréquentés. Nous affirmons donc que l'utilisation du label UNESCO dans la communication touristique relève de la contrainte pour certains acteurs locaux ou du choix pour d'autres. Ceci dépend principalement de leur notoriété. Le label UNESCO peut venir renforcer la notoriété de certains sites culturels ou naturels et les transformer en ressources touristiques.

\section{Conclusion}

Ce travail exploratoire permet de répondre à nos interrogations de départ. L'image d'un territoire joue un rôle important dans le choix d'une destination de séjour touristique. Or, celle-ci s'appuie sur des éléments matériels et sur des éléments idéels. Face à une offre toujours plus importante, le consommateur (futur touriste) peut éprouver des difficultés à trouver sa destination. La notion de risque vient perturber son choix. C'est pourquoi il cherche des signes qui le rassurent et qui vont orienter ses choix. En tant que gage de qualité, les labels jouent un rôle et modifient l'image renvoyée par un territoire.

L'inscription sur la liste du patrimoine mondial, que nous avons considérée comme un label expérientiel tout au long de cette étude, vient peser sur le choix de la destination. En effet, elle met en avant certains sites et donne une image positive aux territoires. Pour l'essentiel des personnes rencontrées, elle est un gage de qualité. Elle peutêtre perçue comme « un chapelet de passages obligés, tous aussi remarquables les uns que les autres, que le voyageur doit expérimenter personnellement» (Brunel, 2006 : 219). En tout état de cause, nous avons montré qu'elle joue un rôle déterminant dans le processus de décision des touristes. Entre deux destinations possibles, le touriste privilégiera celui qui possède un site UNESCO. S'ils connaissent la liste du patrimoine mondial, ils sont peu nombreux à connaitre sa fonction et pensent finalement qu'elle regroupe les sites les plus prestigieux. Ainsi, pour beaucoup, des sites comme ceux de Fontenay, de Saint-Savin-sur-Gartempe ou de Provins n'y figurent pas. Il y a donc indiscutablement un travail d'information et de communication à mener.

Les sites de "second rang» l'ont d'ailleurs bien compris et usent et abusent du label UNESCO dans leur communication
Internet. Il est systématiquement mis en avant et des pages spéciales lui sont consacrées. Les régions et les départements mesurent également l'impact qu'il a et lui accordent une bonne place dans leur communication. À l'inverse, les sites les plus connus comme celui du Mont Saint-Michel l'utilisent très peu. Avec ou sans lui, les touristes seront au rendez-vous tant le site est exceptionnel. Le label est donc indispensable pour les sites peu médiatisés et peu connus, car il dope la fréquentation touristique. Encore faut-il bien communiquer sur ce label! Fontainebleau pourrait sûrement augmenter le nombre de ses visiteurs si le label était mieux mis en avant. Nous insistons donc sur l'importance de communiquer sur ce label qui est un véritable facteur d'attractivité.

Ce travail montre l'intérêt que représente une recherche plus approfondie sur les labels. S'il est vrai que la fréquentation augmente après l'inscription sur la liste du patrimoine mondial, les retombées du label UNESCO sont encore mal évaluées. Il y aurait avantage à déterminer les impacts précisément, de manière à définir la politique de communication la plus adaptée.

\section{Références}

AUDRERIE, Dominique;Raphaël SOUCHIER et Luc VILAR (1998) Le patrimoine mondial,Paris : PUF, coll. Que Sais-Je? 128 p.

BOURDEAU, Laurent et Pascale MARCOTTE (2006) «Valeurs attribuées à un label public par les gestionnaires d'entreprises de services touristiques : le cas d'un label de l'UNESCO».Papier présenté dans le cadre de la $16^{\mathrm{e}}$ conférence internationale RESER (Lisbonne, 28-30 septembre 2006).<www.reser.net/file/28461/>, consulté le 21 novembre 2010. BRUNEL, Sylvie. (2006) La planète disneylandisée, chronique d'un tour du monde, Paris : Éditions Sciences Humaines. 276 p.

CHAMEROY, Fabienne et Jean-Louis CHANDON (2010) «Les labels de qualité ont-ils des effets sur le comportement d'achat du consommateur?». Communication pour le $9^{\mathrm{e}}$ congrès des tendances du marketing en Europe (Venise, 21-23 janvier 2010), 27 p. $<$ http://www.cergam.org/ fileadmin/files/cerog/wp/885.pdf $>$, consulté le 19 novembre 2010.

De GRANDPRÉ, François (2007) «Attraits, attractions et produits touristiques : trois concepts distincts dans le contexte d'un développement touristique régional», Téoros, vol. 26, n 2, p. 12-18.

DE RUYTER, Ko; Martin WETZELSet Mirella KLEIJNEN (2001) "Customer adoption of e-service : an expérimental study», International Journal of Service industry management, vol. 12, nº 2, p. 184-207.

DI MÉO, Guy (1998) Géographie Sociale et territoires, Paris : Éditions Nathan. 317 p.

FROCHOT, Isabelle etPatrick LEGOHÉREL (2007) Le marketing du tourisme, Paris :Dunod. $276 \mathrm{p}$.

FRUSTIER, Pierre (1999) «Pour une évaluation patrimoniale de la communication touristique», Cahier Espace, n 64 (décembre), p. 32-38.

GAGNON, Serge (2007) «Attractivité touristique et "sens" géo-anthropologique des territoires", Téoros, vol. 26, no 2, p. 5-11.

HALL, C. Michaelet Stephen J. PAGE (1999, reprod. 2007) The Geography of Tourism and Recreation: Environment, Place and Space, London :Routledge. 456 p.

JEUGE-MAYNART, Isabelle (dir.) (2010) Dictionnaire Larousse, Paris : Édition Larousse. 3672 p.

KNAFOU, Rémy (1992) «L'invention du Tourisme», DANS Encyclopédie de la géographie,sous la direction de Antoine BAILLY,Robert FERRAS et Denise PUMAIN, p. 851-86. Paris : Economica. 
LARCENEUX, Fabrice (2003a) «Segmentation des signes de qualité : labels expérientiels et labels techniques ", Décisions Marketing, janviermars,vol. 29, p. 35-46.

LARCENEUX, Fabrice (2003b) «Stratégies de signalisation de la qualité : l'impact des labels sur le processus de décision des consommateurs». Thèse de doctorat, Paris : Université de Paris Dauphine. 331 p.

LEIPER, Neil (1990) «Tourist Attraction Systems», Annals of Tourism Research, $\mathrm{n}^{\circ} 17$, p. 367-384.

LEW, Alan A. (1987) «A Framework of Tourist Attraction Research», Annals of Tourism Research, $\mathrm{n}^{\circ}$ 14, p. 553-575.

MACCANNELL, Dean (1989) «Introduction: Semiotic of Tourism», Annals of Tourism Research, ${ }^{\circ} 16$, p. 1-6.

MOUTINHO,Luiz (1987) «Consumer Behavior in Tourism », European Journal of Marketing, vol. 21, ${ }^{\circ} 10$, p. 5-44.

PICOD, Yves (2010) Code de la consommation, Paris : Dalloz. 2040 p

PIKE, Steve(2002) «Destination image analysis : a review of 142 papers from 1973 to 2000 », Tourism Management, vol. 23, n 5, p. 541-549.

PRIM-ALLAZ, Isabelle; Line RICARD; François COURVOISIER; Axel DREYER et Yasmina KHADIR-POGGI (2008) «L'impact des signes de qualité dans le choix d'une destination touristique et dans les choix effectués une fois arrivé à destination». Papier présenté dans le cadre des

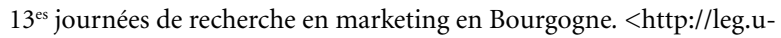
bourgogne.fr/CERMAB/z-outils/documents/actesJRMB/JRMB13-2008/ Prim-Allaz\%20et\%20al.pdf $>$, consulté le 21 novembre 2010.
PROUST, Marcel (1913) Du côté de chez Swann, Paris : Grasset, cité dansDI MÉO, Guy (1998) Géographie Sociale et territoires, Paris : Éditions Nathan. 317 p.

RAFFOUR, Guy (2007) «L'impact et les enjeux du tourisme en ligne», Stratégos, juin à août 2007, <http://www.44news.info/IMG/pdf/E-tourisme_Raffour.pdf $>$, consulté le 18 janvier 2011.

ROBIN Jean-François (2010) directeur de l'Office de tourisme de Provins, entrevue réalisée à Provins, en novembre 2010.

STOCK, Mathis; Olivier DEHOORNE; Philippe Duhamel; Olivier LAZZAROTTI; Rémy KNAFOU, Isabelle SACAREAU et Philippe VIOLIER (2003) Le tourisme. Acteurs, lieux et enjeux, Paris : Belin. 304 p.

UNESCO (1972) «Convention concernant la protection du patrimoine mondial culturel et naturel», Paris : UNESCO. 170 p.

VICTOR, François (2007) La commercialisation des produits et des destinations touristiques : en quoi Internet change-t-il la donne? Étude pour la direction du tourisme, Paris : Kanopée.87p. $<$ http://www.veilleinfotourisme.fr/1179766538343/0/fiche__article/\&RH=RV_strat $>$, consulté le 12 janvier 2011.

WALMSLEY, D. J. et M. YOUNG (1998) «Evaluative image and tourism, the use of personal constructs to describe the structure of destination images», Journal of Travel Research, hiver, p. 65-69.

$\square$ Excellence des destinations
Ciné-tourisme
vol. $30, n^{0} 1,2011$ (15\$)
$\square$ Géotourisme
Tourisme et femmes
vol. $29, n^{0} 2,2010$ (15\$)
Tourisme des racines
Tourisme et autochtones
vol. $29, n^{0} 1,2010$ (15\$)
$\square$ Tourisme, sport et développement
vol. 28, $n^{0} 2,2009$ (15\$)
$\square$ Tourisme polaire
vol. $28, n^{0} 1,2009(15 \$)$

\author{
$\square$ Nouveaux musées, nouveaux tourismes \\ vol. 27, no 3, automne 2008 (12\$) \\ $\square$ Les grands équipements touristiques \\ vol. 27, n 2, été 2008 (12\$) \\ $\square$ La science du tourisme \\ vol. $27, n^{0} 1$, printemps 2008 (12\$) \\ $\square$ Tourisme et solidarité \\ vol. 26, $n^{\circ} 3$, automne 2007 (12\$) \\ $\square$ Tourisme et attractivité \\ vol. 26, no 2, été 2007 (12\$) \\ $\square$ Tourisme Caraïbe \\ vol. 26, $n^{0}$ 1, printemps 2007 (12\$) \\ $\square$ Forêt \\ vol. $25, n^{0} 3$, automne $2006(12 \$)$
}

30 ANS D'EXPÉRIENCE EN TOURISME Complétez votre collection

Retournez ce bon de commande avec votre paiement. Taxes et frais de transport en sus.

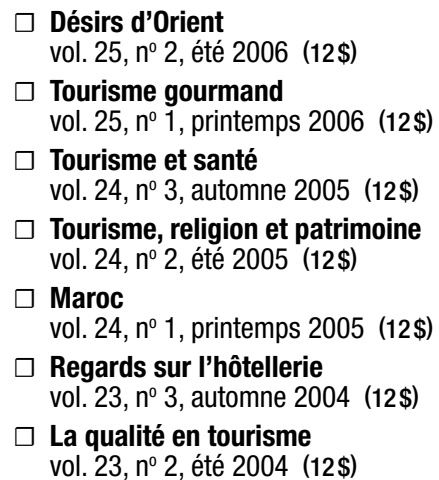

Presses de l'Université du Québec, Service des abonnements Édifice Le Delta I, bureau 450, 2875, boulevard Laurier Québec (Québec) G1V 2M2, CANADA

\begin{tabular}{lll} 
Prénom & Nom & \\
\hline Adresse & Ville & Province \\
\hline Code postal & Pays & \\
\hline Mode de paiement : & $\square$ Chèque (à l'ordre des PUQ) & $\square$ Visa \\
Détenteur de la carte & Numéro de la carte & Master Card \\
\hline Signature & & Date d'expiration
\end{tabular}

